\title{
A Coiflets-Based Wavelet Laplace Method for Solving the Riccati Differential Equations
}

\author{
Xiaomin Wang \\ School of Engineering, Huazhong Agricultural University, Wuhan, Hubei 430070, China \\ Correspondence should be addressed to Xiaomin Wang; wangxm2012@mail.hzau.edu.cn
}

Received 28 January 2014; Accepted 1 July 2014; Published 15 July 2014

Academic Editor: Changbum Chun

Copyright (C) 2014 Xiaomin Wang. This is an open access article distributed under the Creative Commons Attribution License, which permits unrestricted use, distribution, and reproduction in any medium, provided the original work is properly cited.

\begin{abstract}
A wavelet iterative method based on a numerical integration by using the Coiflets orthogonal wavelets for a nonlinear fractional differential equation is proposed. With the help of Laplace transform, the fractional differential equation was converted into equivalent integral equation of convolution type. By using the wavelet approximate scheme of a function, the undesired jump or wiggle phenomenon near the boundary points was avoided and the expansion constants in the approximation of arbitrary nonlinear term of the unknown function can be explicitly expressed in finite terms of the expansion ones of the approximation of the unknown function. Then a numerical integration method for the convolution is presented. As an example, an iterative method which can solve the singular nonlinear fractional Riccati equations is proposed. Numerical results are performed to show the efficiency of the method proposed.
\end{abstract}

\section{Introduction}

In the recent years, fractional differential equations have been found to be effective to describe some physical phenomena such as rheology, damping laws, fractional random walk, and fluid flow [1-6]; the analytical asymptotic techniques of solutions to various fractional differential equations have been studied and many new numerical techniques have been widely applied to the nonlinear problems. In this paper, we present numerical solutions for the fractional Riccati differential equation

$$
\begin{array}{r}
D^{\alpha} y(t)=A(t)+B y(t)+C[y(t)]^{2}, \\
t>0, \quad w-1<\alpha_{m} \leq w
\end{array}
$$

with initial conditions

$$
D_{t}^{\lambda} y(0)=y_{\lambda}, \quad \lambda=0,1, \ldots, w-1,
$$

where $A(t)$ is a known function, $B, C, y_{\lambda}, \lambda=0,1, \ldots, w-$ 1 , are arbitrary constants, and $\alpha$ is a parameter describing the order of the fractional derivative. The general response expression contains a parameter describing the order of the fractional derivative that can be varied to obtain various responses. For the case of $\alpha=1$, the fractional equation reduces the classical Riccati differential equation.

In this paper, we will introduce a modified fractional differential operator $D^{\alpha}$ proposed by Caputo and Mainardi [3]. We mention the important example: the Riccati equations, which play a significant role in many fields of engineering and applied science $[1,5]$, and the analytical solution for Riccati equation in an explicit form seems to be unlikely except for certain special situations. Therefore, the numerical solutions for the Riccati differential equations are important for engineers and scientists. For example, Mohammadi and Hosseini [5] and Momani and Shawagfeh [7] solved nonlinear partial differential equations of fractional order by the Adomian decomposition method (ADM), which is based on applying the inverse of the fractional operator and has complicated algorithms in calculating much Adomian polynomials for nonlinear problems, and the He's variational iteration method (VIM) [8], the piecewise variational iteration method (VIM) [9], which does not need the so-called Adomian polynomials but depends on variational theory with the proper selection of the initial approximation $u_{0}(t)$, and they both need a large number of iterative calculations. The homotopy perturbation 
(HPM) $[10,11]$ provides an effective procedure for explicit and numerical solutions of differential equations; however, the rate of convergence of the series solution depends on the auxiliary parameter $h$ and an initial guess $u_{0}(t)$.

Despite the progresses outlined above, the literatures on high accuracy and easy to implement numerical techniques are suitable for solving nonlinear Riccati differential equations. The wavelet method, which is a general analytical method and has been widely used by many mathematicians and engineers to solve various functional equations, was applied to signal decompositions and reconstructions, Laplace inversions [12], differential equation solutions [13$15]$, and active vibration control of piezoelectric smart structures $[16,17]$. However, it is somewhat surprising that only very few studies have focused on the solution of fractional differential equations by using wavelet methods $[18,19]$.

In this paper, we introduce a Coiflets-based wavelet Laplace method (CWLM) that can efficiently solve Riccati differential equation. This method depends on an explicit wavelet approximation scheme for the nonlinear terms of unknown function in the equation, in which series coefficients are just the function samplings at corresponding nodal points, and also by using Laplace transform, the equation with singular integral kernel was converted into equivalent nonsingular integral equation. At last, numerical simulations are performed to show the efficiency of the method proposed.

\section{Preliminaries}

2.1. Fractional Derivative Operator. Here we give some necessary definitions and mathematical preliminaries of the fractional calculus which are used in this paper. The two most commonly used definitions are the Riemann-Liouville and Caputo. The difference between the two definitions is in the order of evaluation. The Riemann-Liouville fractional derivative operator of order $\alpha>0$ is defined as [2]

$$
D_{t}^{\alpha} f(t)=\frac{1}{\Gamma(\alpha)} \int_{0}^{t}(t-\tau)^{\alpha-1} f(\tau) d \tau, \quad t>0 .
$$

Since the Riemann-Liouville fractional derivative has some disadvantages when trying to model real-world phenomena with initial and boundary conditions of fractional differential equations, in this paper, we will introduce a modified fractional differential operator which is proposed by Caputo and Mainardi [3].

For $n$ to be the smallest integer that exceeds $\alpha$, the Caputo time-fractional derivative operator of order $\alpha>0$ is defined as [3]

$$
D_{t}^{\alpha} f(t)= \begin{cases}f^{(n)}(t), & \alpha=n \in N \\ \frac{1}{\Gamma(n-\alpha)} \int_{0}^{t} \frac{f^{(n)}(\tau)}{(t-\tau)^{\alpha+1-n}} d \tau, & n-1<\alpha<n,\end{cases}
$$

where $f^{(n)}(t)$ is $n$-order derivative of the function $f(t)$ and $\Gamma(n-\alpha)$ is the Gamma function.
The Laplace transform of Caputo fractional derivative operator $D_{t}^{\alpha} f(t)$ is [3]

$$
\begin{array}{r}
L\left[D_{t}^{\alpha} f(t)\right]=s^{\alpha} L[f(t)]-\sum_{m=0}^{n-1} s^{\alpha-1-m} f^{(m)}\left(0^{+}\right), \\
n-1<\alpha \leq n, \quad n \in N .
\end{array}
$$

For more details on fractional calculus see $[2,3]$.

2.2. Coiflets Orthogonality Wavelet. Function $\phi(x) \in L^{2}(\mathbf{R})$ is called orthogonal scaling function, if $\phi(x)$ has the following properties.

(1) There is a sequence set $\left\{p_{k}, k \in \mathbf{Z}\right\}$, and it satisfies

$$
\phi(x)=\sum_{k} p_{k} \phi(2 x-k) .
$$

(2) Set $\left\{\phi_{n, k}(x)=2^{n / 2} \phi\left(2^{n} x-k\right), k \in \mathbf{Z}\right\}$. Satisfy the orthogonality conditions; that is,

$$
\int_{-\infty}^{\infty} \phi_{n, k}(x) \phi_{n, l}(x) d x=\delta_{k, l}
$$

where

$$
\delta_{k, l}= \begin{cases}0, & k \neq l \\ 1, & k=l\end{cases}
$$

is Kronecker-Delta function.

(3) For all $f \in L^{2}(\mathbf{R})$, note

$$
\mathbf{P}_{n} f(x) \equiv \sum_{k \in \mathbf{Z}} c_{n, k} \phi_{n, k}(x)
$$

where

$$
c_{n, k} \equiv \int_{\mathbf{R}} f(x) \phi_{n, k}(x) d x
$$

Function space is

$$
V_{n} \equiv \cos _{L}^{2}(\mathbf{R})\left\{\phi_{n, k}\right\}, \quad k \in Z>, n \in Z
$$

Satisfy the following relations:

(i) $\{0\} \cdots \subset V_{-2} \subset V_{-1} \subset V_{0} \subset V_{1} \subset \cdots \subset L^{2}(\mathbf{R})$;

(ii) $\mathbf{P}_{n} f(x) \in V_{n} \leftrightarrow \mathbf{P}_{n} f(2 x) \in V_{n+1}$;

(iii) $\mathbf{P}_{n} f(x) \in V_{n} \leftrightarrow \mathbf{P}_{n} f\left(x+2^{-n}\right) \in V_{n}$;

(iv) $\lim _{n \rightarrow \infty} V_{n}=\cup_{n} V_{n}$, is dense in $L^{2}(\mathbf{R})$;

(v) $\lim _{n \rightarrow \infty} \cap_{n} V_{n}=\{0\}$;

(vi) setting $\{\phi(x-k)\}$ forming a Riese base of $V_{0}$; that is, there exist constants $A$ and $B(0<A \leq B<\infty)$, satisfy

$$
A \sum_{k \in \mathbf{Z}}\left|c_{k}\right|^{2} \leq\left\|\sum_{k \in \mathbf{Z}} c_{k} \phi(x-k)\right\|_{2}^{2} \leq B \sum_{k \in \mathbf{Z}}\left|c_{k}\right|^{2} .
$$


For the square sum of sequence space $l_{2}$, arbitrary sequence $\left\{c_{k}\right\} \in l_{2}$. Where coefficient $p_{k}$ is low-pass filter coefficient, it usually only has a finite number of nonzero value.

For any function $f(x) \in L^{2}(\mathbf{R})$, we have the following approximation:

$$
f(x) \approx \mathbf{P}_{n} f(x)=\sum_{k=-\infty}^{\infty} c_{n, k} \phi_{n, k}(x),
$$

where the coefficient

$$
c_{n, k}=\int_{-\infty}^{\infty} f(x) \phi_{n, k}(x) d x .
$$

For the Coiflets wavelet scaling function, Zhou and Wang [20] have proposed the generalized Gaussian integral method of wavelet, then we have

$$
c_{n, k}=\int_{-\infty}^{\infty} f(x) \phi_{n, k}(x) d x \approx 2^{-n / 2} f\left(\frac{M_{1}+k}{2^{n}}\right),
$$

where $M_{1}=\int_{-\infty}^{+\infty} x \phi(x) d x$ is the first-order moment of scaling function, which can be obtained accurately by filter coefficients in the two-scale equations. The approximation accuracy of (13) depends on the corresponding wavelet function vanishing moments, when the corresponding scaling function $\phi_{n, k}(x)$ has the order of vanishing moment $\gamma$, and $f(x) \in C^{\gamma}$, and we have [20]

$$
\left\|f(x)-\mathbf{P}_{n} f(x)\right\|=O\left(2^{-n \gamma}\right) .
$$

It means that the error of (13) becomes smaller while the scale and the maximum order of vanishing moments of scaling function approximation increase. Substitute (15) into (13)

$$
f(x) \approx \sum_{k=-\infty}^{\infty} f\left(\frac{M_{1}+k}{2^{n}}\right) \phi\left(2^{n} x-k\right) .
$$

The form equation (17) of the function of single-point reconstruction formula has the following characteristics [20].

(1) For the composite function of the function $f(x)$ or the functional operations $\Pi[f(x)]$, its reconstruction (or approximation) formula is

$$
\Pi f(x) \approx 2^{-n / 2} \sum_{k=-\infty}^{\infty} \Pi f\left(\frac{M_{1}+k}{2^{n}}\right) \phi_{n, k}(x),
$$

where $\phi_{n, k}(x)=2^{n / 2} \phi\left(2^{n} x-k\right)$; the establishment of (18) is obvious, because (18) is for the establishment of an arbitrary function form, of course, including its complex functional form. Therefore, simply square integrable complex functions $\Pi[f(\cdot)]$ can be treated as $f(\cdot)$ in (18). In this way, furthermore, the importance of (18) is, for an arbitrary function $f(x)$ over the function operator $\Pi$ (which may be nonlinear operator) transformation, the computing of its right-hand side is equivalent to a linear operator computing. That is, the coefficient of the scaling function $\Pi[f(x)]$ can be obtained by put operator $\Pi$ on the role of the coefficients $f(\cdot)$.
(2) For the integral operator or the derivative operator $\mathbf{D}$ on the function $f(x)$, we have

$$
\mathbf{D}[f(x)] \approx 2^{-n / 2} \sum_{k=-\infty}^{\infty} f\left(\frac{M_{1}+k}{2^{n}}\right) \mathbf{D}\left[\phi_{n, k}(x)\right] .
$$

That is, we just need to put the role of operator $\mathbf{D}$ on $\phi_{n, k}(x)$ in (17).

As we know, wavelet series approximation is a square integrable function defined in the infinite interval, when the approximating function is defined only in a finite interval, and we need to truncate the wavelet series, which may introduce the boundary effect significantly, and the corresponding numerical method of calculation led to decreased accuracy. Traditionally, general treatment of boundary conditions is by using the zero-extension, symmetric, or periodic extension and so on. To some extent, these approaches can effectively inhibit the jitter of the border when it is a special form of approximation function, but not universal, and does not consider the wavelet expansion to meet the boundary conditions. Different from the past expansion of function, in this paper, based on Taylor series expansion of the boundary extension which is applied on the function defined on a finite interval $[14,15]$, then the function needs to satisfy the corresponding boundary conditions which is embedded in the corresponding Taylor series.

First, we assume that the function $g(x) \in L^{2}[0, b]$, using Taylor series expansion on the borders, forms as follows:

$$
g(x)= \begin{cases}\sum_{i=0}^{M} \frac{1}{i !} \frac{d^{i} g(0)}{d x^{i}} x^{i} & x \in(-\infty, 0) \\ g(x) & x \in[0, b] \\ \sum_{i=0}^{M} \frac{1}{i !} \frac{d^{i} g(b)}{d x^{i}}(x-b)^{i} & x \in(b, \infty),\end{cases}
$$

where $\delta>0$ and $\left(d^{i} g(0) / d x^{i}\right)=\sum_{k=0}^{\alpha} p_{0, i, k} g\left(k / 2^{n}\right)$, $\left(d^{i} g(b) / d x^{i}\right)=\sum_{k=0}^{\alpha} p_{b, i, k} g\left(b-\left(k / 2^{n}\right)\right), i=0,1,2, \ldots$, are expressed by the numerical difference from the interior point, and $p_{0, i, k}, p_{b, i, k}$ are the numerical difference coefficients. When $m=3, M=3$, according to four-point-Malkoff numerical difference formulas $[14,15]$, we know

$$
\begin{aligned}
& \mathbf{A}_{0}=\left(\begin{array}{cccc}
1 & 0 & 0 & 0 \\
-\frac{11}{6} & 3 & -\frac{3}{2} & \frac{1}{3} \\
2 & -5 & 4 & -1 \\
-1 & 3 & -3 & 1
\end{array}\right), \\
& \mathbf{A}_{1}=\left(\begin{array}{cccc}
1 & 0 & 0 & 0 \\
\frac{11}{6} & -3 & \frac{3}{2} & -\frac{1}{3} \\
2 & -5 & 4 & -1 \\
1 & -3 & 3 & -1
\end{array}\right),
\end{aligned}
$$

where $A_{0}=\left\{2^{-i n} p_{0, i, k}\right\}$ and $A_{1}=\left\{2^{-i n} p_{b, i, k}\right\}(i, k=0,1,2,3)$. For the specific boundary conditions, once the boundary conditions are given in (21), certain elements of the matrix 
values will be adjusted. For example, for boundary conditions $x=0,\left(d^{i} g(0) / d x^{i}\right)=0$, we just need to make $p_{0, i, k}=0$, $(k=0,1,2,3)$, and other $p_{0, j, k}(j \neq i)$ take the values given in (21) without change.

Then, (18) can be expressed as

$$
g(x)= \begin{cases}\sum_{k=0}^{3} g\left(\frac{k}{2^{n}}\right) T_{0, k}(x), & x \in(-\infty, 0) \\ g(x), & x \in[0, b] \\ \sum_{k=0}^{3} g\left(b-\frac{k}{2^{n}}\right) T_{b, k}(x), & x \in(b, \infty),\end{cases}
$$

where $T_{0, k}(x)=\sum_{i=0}^{M}\left(p_{0, i, k} / i !\right) x^{i}, T_{b, k}=\sum_{i=0}^{M}\left(p_{b, i, k} / i !\right)(x-b)^{i}$, and $2^{n} \geq m$.

Consider Coiflets scaling function $\phi(x)$ with the support set $[0, N-1], N=3 \gamma$, applying the scaling function series approximation equation (17), and $M_{1}$ is an integral; for the function $g(x) \in L^{2}[0, b]$, we have the following form:

$$
g(x) \approx \sum_{k=2-3 N+M_{1}}^{b 2^{n}+M_{1}-1} g\left(\frac{k}{2^{n}}\right) \phi\left(2^{n} x+M_{1}-k\right) .
$$

Substituting (22) into (23), then (23) can be rewritten as

$$
g(x) \approx \sum_{k=0}^{b 2^{n}} g_{k} \Phi_{b, n, k}(x)
$$

where $g_{k}=g\left(k / 2^{n}\right)$, and we denote

$\Phi_{b, n, k}(x)$

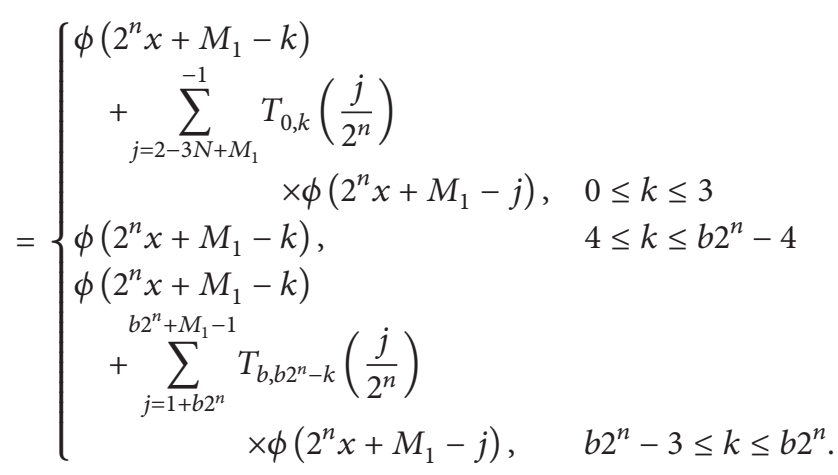

Thus, when specific boundary conditions are given, the differential coefficients of extension can be determined in accordance with the above process, and the corresponding improved scaling function is given by (25).

\section{Numerical Algorithm}

In this section, we will consider the modified wavelet approximation scheme to solve nonlinear Riccati differential equation (1) with the initial condition expressed by (2).
First, applying the Laplace transform on the time variable $t$ in (1) leads to

$$
s^{\alpha} Y(s)=A(s)+B Y(s)+C L\left[(y(t))^{2}\right]+\sum_{\lambda=0}^{[\alpha]} s^{\alpha-1-\lambda} y_{\lambda}
$$

that is,

$$
Y(s)=s^{p} R(s)\left\{A(s)+C L\left[(y(t))^{2}\right]\right\}+\sum_{\lambda=0}^{[\alpha]} s^{\alpha-1-\lambda} y_{\lambda} s^{p} R(s)
$$

in which $R(s)=1 / s^{p}\left(s^{\alpha}-B\right) \cdot p=0$ or $p=1$, and the formula of the Laplace transform of the fractional order derivative is considered [3]. When the function obtained by the inverse Laplace transform of $R(s)$ is singular, we take $p=1$, else we take $p=0$. For example, for $B=0,0<\alpha \leq 1$, we just need to take $p=1$; then $R(s)=1 / s^{\alpha+1}$, whose inverse Laplace transform is $t^{\alpha} / \operatorname{gamma}(\alpha+1)$, and it is nonsingular. Taking the inverse Laplace transform of (27), we obtain

$$
y(t)=\int_{0}^{t} r(t-\tau) \frac{\partial^{p}}{\partial \tau^{p}}\left[C(y(\tau))^{2}+A(\tau)\right] d \tau+\sum_{\lambda=0}^{[\alpha]} y_{\lambda} r_{\lambda}(t),
$$

where

$$
r(t)=L^{-1}(R(s)), \quad r_{\lambda}(t)=L^{-1}\left(s^{\alpha-1-\lambda} s^{p} R(s)\right) .
$$

By [12], $r(t), r_{\lambda}(t)$ can be obtained by numerical method. From [6], we know

$$
L^{-1}\left[\frac{s^{\alpha-\beta}}{s^{\alpha}+\sigma}\right]=t^{\alpha+\beta-1} E_{\alpha, \beta}\left(\sigma t^{\alpha}\right)
$$

where $E_{\alpha, \beta}\left(\sigma t^{\alpha}\right)$ is the generalized Mittag-Leffler type functions defined in the power series

$$
E_{a, b}(z)=\sum_{k=0}^{\infty} \frac{z^{k}}{\Gamma(a k+b)}
$$

It can be seen from (30) that the integral kernel $r(t)$ is a nonsingular smooth function with property of $r(0)=0$ when $0<\alpha \leq 2$; applying (24), (25), we have

$$
y(t) \approx \sum_{k=0}^{b 2^{n}} y\left(t_{k}\right) \Phi_{b, n, k}(t) .
$$

By using (19), we denote

$$
\begin{aligned}
H^{(p)}(t) & =\frac{\partial^{p}}{\partial t^{p}}\left[C(y(\tau))^{2}+A(\tau)\right] \\
& \approx \sum_{j=0}^{b 2^{n}}\left[C\left(y\left(t_{j}\right)\right)^{2}+A\left(t_{j}\right)\right] \Phi_{b, n, j}^{(p)}(t) .
\end{aligned}
$$


Applying (24) to approximate the term $r(t-\tau) H^{(P)}(\tau)$ with $\tau \in[0, t]$ in (28), we have

$$
r(t-\tau) H^{(P)}(\tau) \approx \sum_{k=0}^{t 2^{n}} r\left(t-t_{k}\right) H^{(P)}\left(t_{k}\right) \Phi_{t, n, k}(\tau),
$$

where $t_{k}=k / 2^{n}$. Integrating both sides of (34), we obtain

$$
\int_{0}^{t} r(t-\tau) H^{(P)}(\tau) d \tau \approx \sum_{k=0}^{t 2^{n}} r\left(t-t_{k}\right) H^{(P)}\left(t_{k}\right) \Phi_{t, n, k}^{\int}(t),
$$

where $\Phi_{t, n, k}^{\int}\left(t_{i}\right) \equiv \int_{0}^{t_{i}} \Phi_{t, n, k}(\tau) d \tau$ can be exactly obtained according to Wang [22]. Inserting (35) into (28) and then setting $t=t_{i}$ yield

$$
y_{i} \approx \sum_{k=0}^{i} r_{i-k} H^{(P)}\left(t_{k}\right) \Phi_{t_{i}, n, k}^{\int}\left(t_{i}\right),
$$

where $y_{i}=y\left(t_{i}\right), t_{i}=i / 2^{n}(i=1,2,3, \ldots)$ and $r_{i-k}=r\left(t_{i}-\right.$ $\left.t_{k}\right)$. Then one can solve the algebraic equations to obtain the solution of the Riccati equation. According to (30), note that if there should be $r(0)=0$ for $0<\alpha \leq 2$, which implies that $r(i-k)\left[g\left(y_{k}\right)-f_{k}\right] \Phi_{t_{i}, n, k}^{\int}\left(t_{i}\right)=0$ when $k=i$, thus (36) can be further simplified into the form

$$
y_{i} \approx \sum_{k=0}^{i-1} r_{i-k} H^{(P)}\left(t_{k}\right) \Phi_{t_{i}, n, k}^{J}\left(t_{i}\right)
$$

It can be seen from (37) that the solution $y_{i}$ can be directly obtained step-by-step as the index $i$ increases. In this process, no matrix inversion is needed. The scale $n$ is bigger, and the precision of the results of (36) and (37) is better, which will be shown in examples. In fact, in most practice problems, (37) is possible, and even without the special nature of $r(t)$ in some case, we can still solve the nonlinear algebraic equation (36), which is nonsingular and easy. Then we promote the method to the fractional Riccati equations.

\section{Numerical Examples}

In this section we will give two numerical experimentsto illustrate the efficiency and apply the approach proposed in this paper.

Example 1. Consider the following fractional Riccati equation given in $[7,19,21]$ :

$$
D^{\alpha} y(t)=1-[y(t)]^{2}, \quad t>0,0<\alpha \leq 1
$$

with initial conditions

$$
y(0)=0, \quad(0<\alpha \leq 1)
$$

and the exact solution of (38) is $y(t)=\left(e^{2 t}-1\right) /\left(e^{2 t}+1\right)$ when $\alpha=1$.

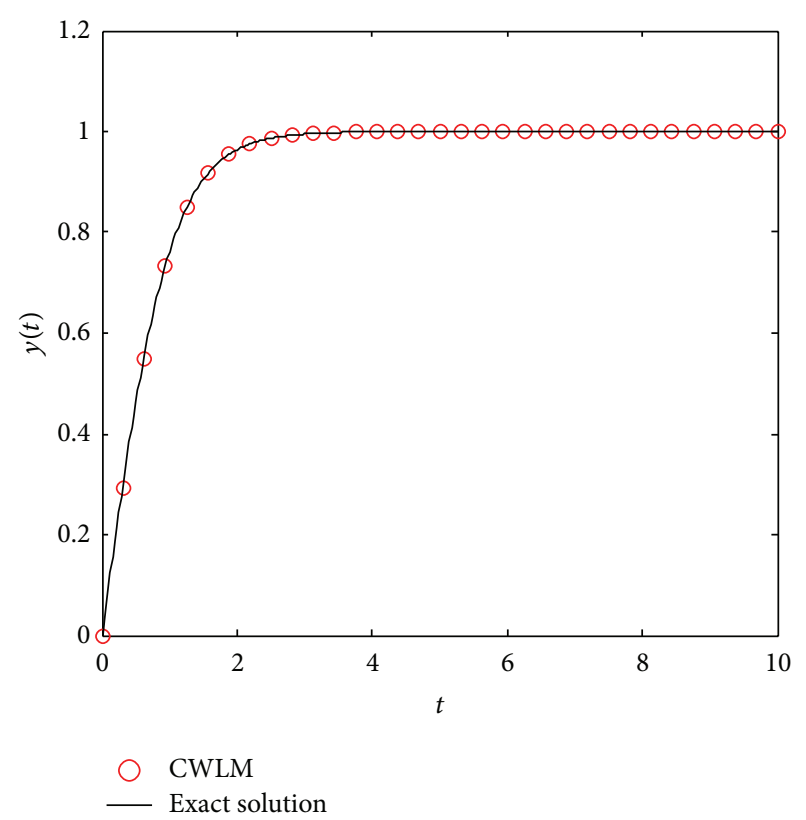

FIGURE 1: Comparison between the numerical and exact solution of Example 1 for $\alpha=1$.

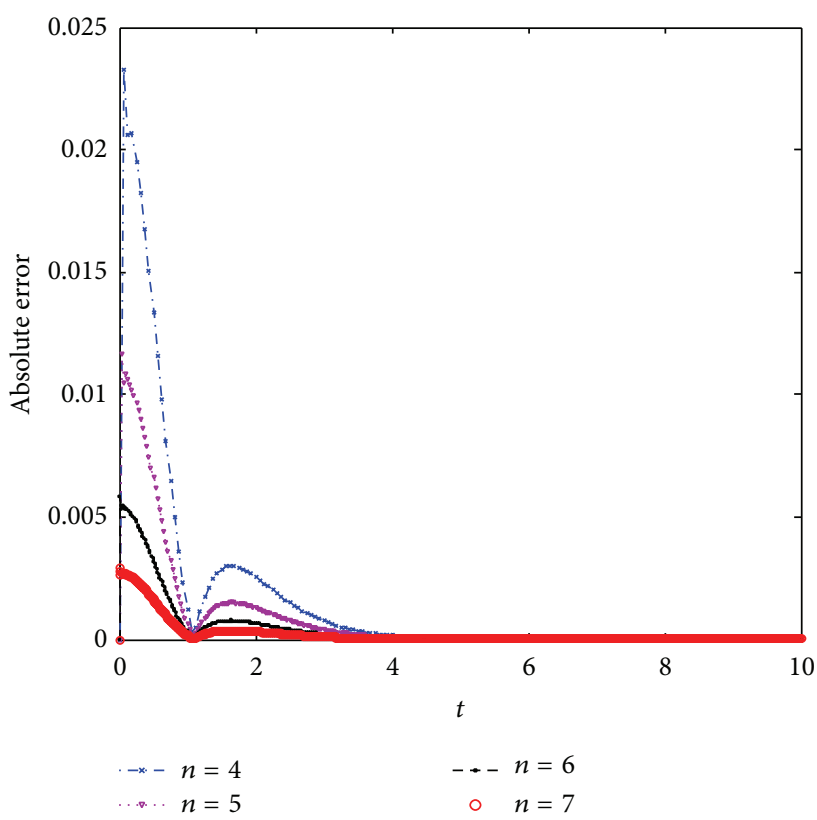

Figure 2: The absolute errors of the exact solution and the results obtained by CWLM with different $n$ for $\alpha=1$.

Figure 1 is the comparison between the exact result and the numerical result for $n=5$. We can see that the numerical solution is in very good agreement with the exact solution. Figure 2 shows the absolute errors of the exact solution and the results obtained by CWLM with different $n$. One can see that approximate solution converges to the exact solution, and the absolute error goes down while $n$ is increased. Contrast of numerical solution of (38) for $\alpha=0.5$ and $\alpha=1$ is given in Figure 3. 


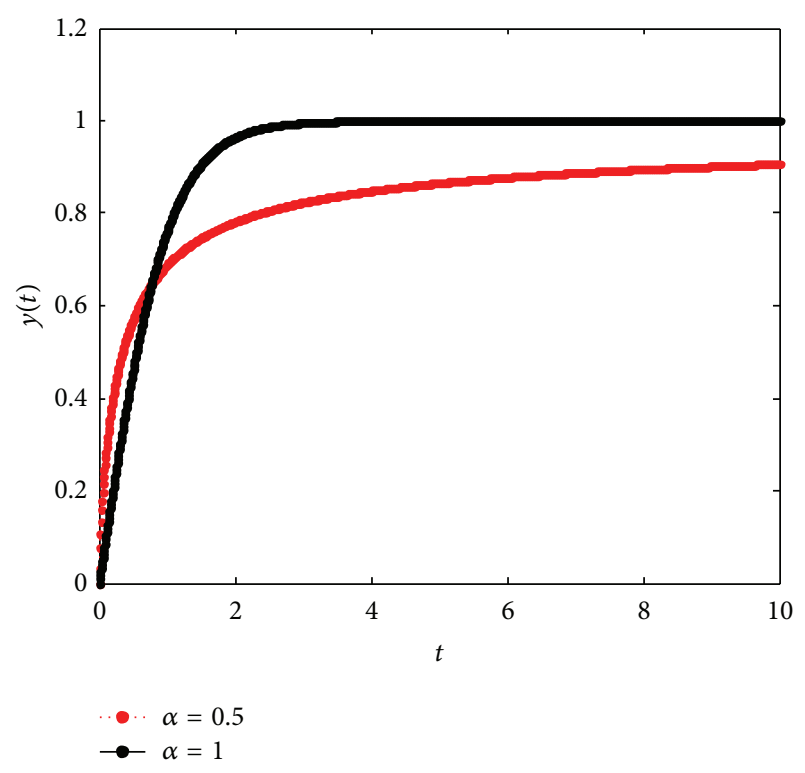

Figure 3: Solution of (38) obtained by the CWLM for $\alpha=0.5$ and $\alpha=1$.

TABLE 1: Solution of (38) obtained by different methods for $\alpha=0.5$.

\begin{tabular}{lccccc}
\hline \multicolumn{5}{c}{ Methods } \\
$t$ & HAM [21] & HWOMM [19] & & CWLM \\
& & & $n=4$ & $n=6$ & $n=7$ \\
\hline 1.0 & 0.6982 & 0.6987 & 0.6667 & 0.6837 & 0.6883 \\
2.0 & 0.7858 & 0.7857 & 0.7732 & 0.7796 & 0.7814 \\
3.0 & 0.8268 & 0.8258 & 0.8190 & 0.8224 & 0.8234 \\
4.0 & 0.8525 & 0.8499 & 0.8455 & 0.8477 & 0.8484 \\
5.0 & 0.8714 & 0.8664 & 0.8633 & 0.8648 & 0.8652 \\
6.0 & 0.8869 & 0.8785 & 0.8761 & 0.8773 & 0.8776 \\
7.0 & 0.9004 & 0.8878 & 0.8860 & 0.8869 & 0.8871 \\
8.0 & 0.9127 & 0.8953 & 0.8938 & 0.8946 & 0.8948 \\
9.0 & 0.9243 & 0.9016 & 0.9003 & 0.9009 & 0.9011 \\
10.0 & 0.9354 & 0.9068 & 0.9057 & 0.9062 & 0.9064 \\
\hline
\end{tabular}

Table 1 is the solutions of Example 1 obtained by different methods when $\alpha=0.5$. Compared with results in [21] obtained by using HAM with $[4,4]$ homotopy-Pade approximations and HWOMM in [19], which need to solve a nonlinear system of nearly hundreds of algebraic equations.

Example 2. Consider the following fractional Riccati equation $[7,19]$ :

$$
D^{\alpha} y(t)=1+2 y(t)-[y(t)]^{2}, \quad t>0,0<\alpha \leq 1 .
$$

With initial conditions

$$
y(0)=0
$$

the exact solution is $y(t)=1+\sqrt{2} \tanh (\sqrt{2} t+(1 / 2) \log ((\sqrt{2}-$ 1) $/(\sqrt{2}+1)))$ when $\alpha=1$.

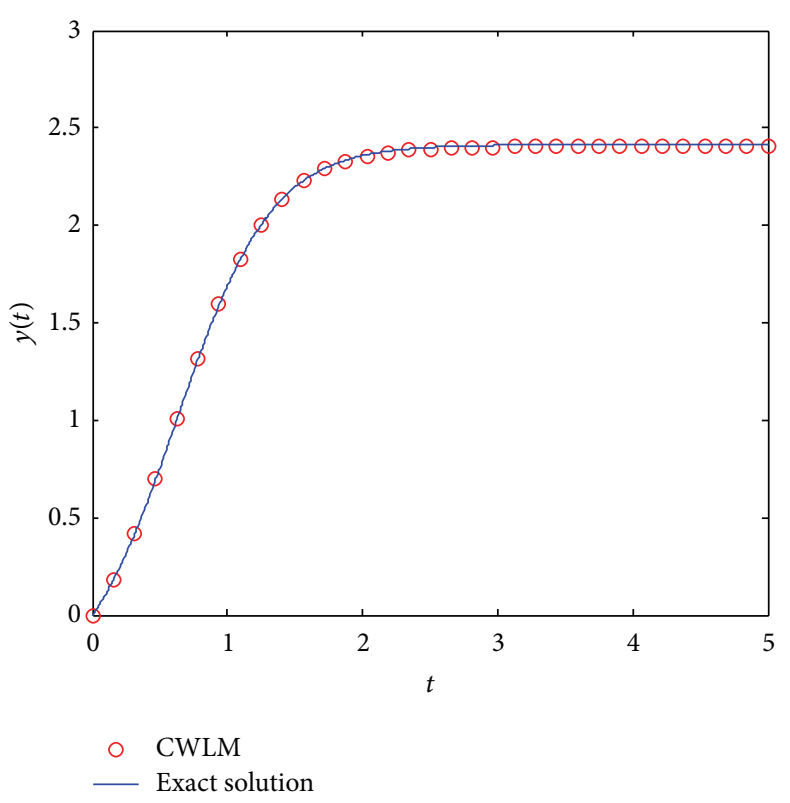

FIGURE 4: Comparison between the numerical and exact solutions of Example 2 for $\alpha=1$.

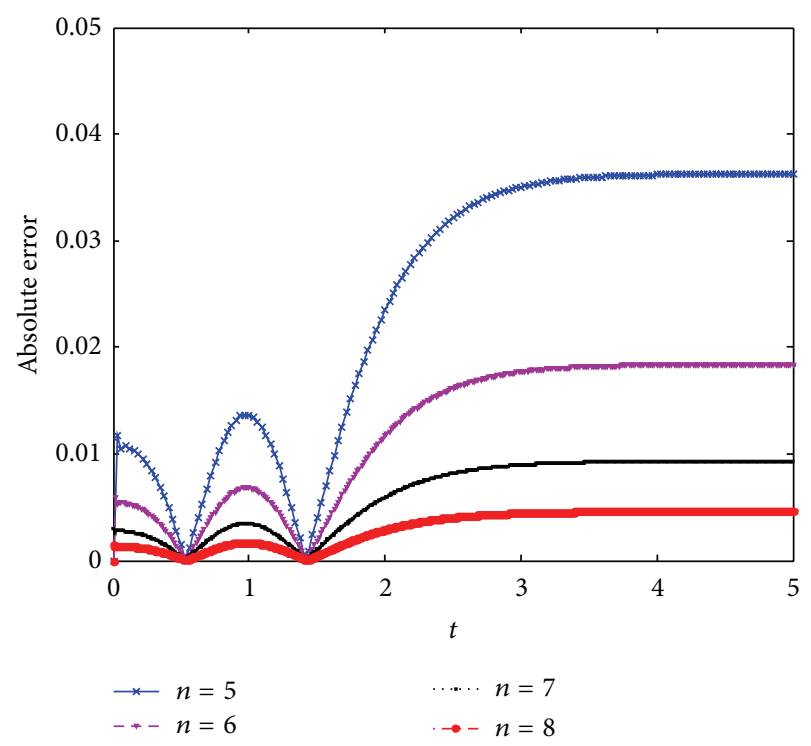

Figure 5: The absolute errors of the exact solution and the results obtained by CWLM with different $n$ for $\alpha=1$.

Figure 4 is the comparison between the exact result and the numerical result for $n=7$. One can see that CWLM is accurate and is able to solve this nonlinear Riccati differential equation in a very wider region. Figure 5 shows the absolute errors of the exact solution and the results obtained by CWLM with different $n$ for Example 2 when $\alpha=1$, in which it can be seen that the bigger the value of the scale is, the smaller absolute error is. Figure 6 is the solution of (40) obtained by the CWLM for $\alpha=0.75$ and $\alpha=1$, compared with results obtained by other methods in $[19,23,24]$, which are given in Table 2. 


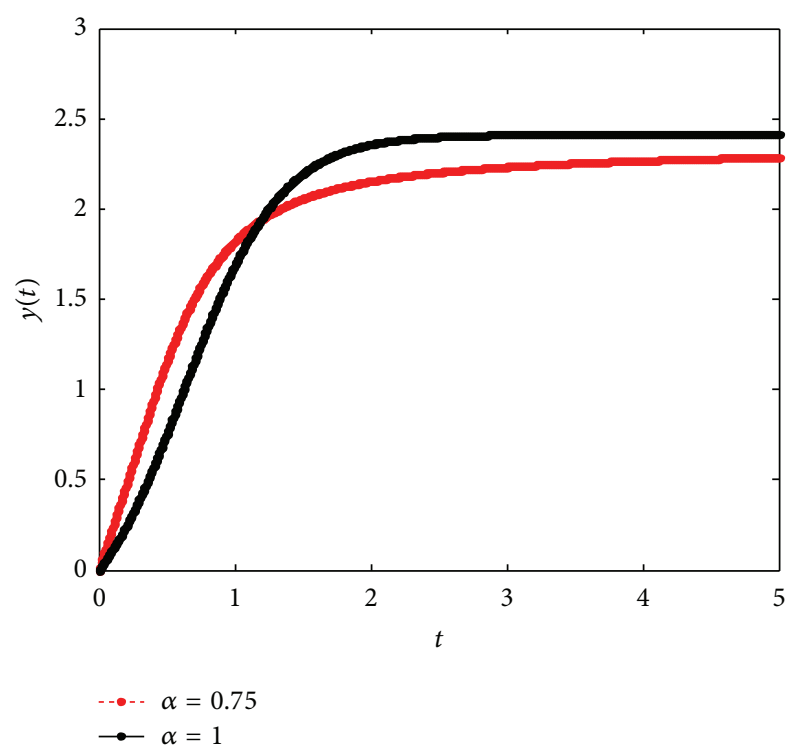

Figure 6: Solution of (40) obtained by the CWLM for $\alpha=0.75$ and $\alpha=1$.

TABle 2: Solution of (40) obtained by the EHPM, MHPM, HWOMM, and CWLM for $\alpha=0.75$.

\begin{tabular}{lccccc}
\hline \multicolumn{7}{c}{ Methods } \\
$t$ & EHPM [23] & MHPM [24] & HWOMM [19] & \multicolumn{2}{c}{ CWLM } \\
& & & & $n=7$ & $n=8$ \\
\hline 1.0 & 1.9753 & 2.0874 & 1.8186 & 1.1571 & 1.1538 \\
2.0 & 2.2062 & 0.7787 & 2.1796 & 1.8206 & 1.8198 \\
3.0 & 2.2813 & 5.8102 & 2.2684 & 2.0566 & 2.0629 \\
4.0 & 2.3153 & -0.0807 & 2.3062 & 2.1539 & 2.1643 \\
5.0 & 2.3340 & -396.4145 & 2.3273 & 2.2038 & 2.2164 \\
6.0 & 2.3460 & $-2.8182 e 3$ & 2.3408 & 2.2337 & 2.2477 \\
7.0 & 2.3544 & $-1.1876 e 4$ & 2.3504 & 2.2537 & 2.2685 \\
8.0 & 2.3608 & $-3.7971 e 4$ & 2.3574 & 2.2679 & 2.2833 \\
9.0 & 2.3657 & $-1.0148 e 5$ & 2.3629 & 2.2787 & 2.2945 \\
10.0 & 2.3679 & $-2.3858 e 5$ & 2.3673 & 2.2870 & 2.3031 \\
\hline
\end{tabular}

\section{Conclusions}

In this paper, a numerical method based on the Coiflets wavelet operational method is applied to solve the fractional differential equations. In this method, the equation with fractional differential order is transferred to an integral equation of convolution type by the Laplace transform and then the solution is approximated by the modified wavelet approximate scheme. This simple method was established by Zhou et al. [14] (including the author) and has been applied to solve nonlinear equations of vibration, diffusion, and wave equations. Compared with the results for solving fractional Riccati differential equation by the other numerical methods [7, 19, 21, 23, 24], the results for numerical examples demonstrate that the present method can give a high accurate approximation in a larger region. This is also the advantage of the present method.

\section{Conflict of Interests}

The author declares that there is no conflict of interests regarding the publication of this paper.

\section{Acknowledgments}

This work was supported by National Natural Science Foundation of China (11302081) and Huazhong Agricultural University Scientific and Technological Self-innovation Foundation under Grant (52902-0900206074).

\section{References}

[1] W. T. Reid, Riccati Differential Equations, Academic Press, New York, NY, USA, 1972.

[2] I. Podlubny, Fractional Differential Equations, Academic Press, San Diego, Calif, USA, 1999.

[3] M. Caputo and F. Mainardi, "Linear models of dissipation in anelastic solids," La Rivista del Nuovo Cimento, vol. 1, no. 2, pp. 161-198, 1971.

[4] R. Metzler, E. Barkai, and J. Klafter, "Anomalous diffusion and relaxation close to thermal equilibrium: a fractional FokkerPlanck equation approach," Physical Review Letters, vol. 82, no. 18 , pp. 3563-3567, 1999.

[5] F. Mohammadi and M. M. Hosseini, "A comparative study of numerical methods for solving quadratic Riccati differential equations," Journal of the Franklin Institute, vol. 348, no. 2, pp. 156-164, 2011.

[6] D. Hong, Y. Kim, and J. Wang, "A new approach for the analysis solution of dynamic systems containing fractional derivative," Journal of Mechanical Science and Technology, vol. 20, no. 5, pp. 658-667, 2006.

[7] S. Momani and N. Shawagfeh, "Decomposition method for solving fractional Riccati differential equations," Applied Mathematics and Computation, vol. 182, no. 2, pp. 1083-1092, 2006.

[8] S. Abbasbandy, "A new application of He's variational iteration method for quadratic Riccati differential equation by using Adomian's polynomials," Journal of Computational and Applied Mathematics, vol. 207, no. 1, pp. 59-63, 2007.

[9] F. Geng, Y. Lin, and M. Cui, "A piecewise variational iteration method for Riccati differential equations," Computers \& Mathematics with Applications, vol. 58, no. 11-12, pp. 2518-2522, 2009.

[10] S. Abbasbandy, "Homotopy perturbation method for quadratic Riccati differential equation and comparison with Adomian's decomposition method," Applied Mathematics and Computation, vol. 172, no. 1, pp. 485-490, 2006.

[11] S. Abbasbandy, "Iterated He's homotopy perturbation method for quadratic Riccati differential equation," Applied Mathematics and Computation, vol. 175, no. 1, pp. 581-589, 2006.

[12] J. Wang, Y. Zhou, and H. Gao, "Computation of the Laplace inverse transform by application of the wavelet theory," Communications in Numerical Methods in Engineering, vol. 19, no. 12, pp. 959-975, 2003.

[13] Y. Li, "Solving a nonlinear fractional differential equation using Chebyshev wavelets," Communications in Nonlinear Science and Numerical Simulation, vol. 15, no. 9, pp. 2284-2292, 2010.

[14] Y. H. Zhou, X. M. Wang, J. Z. Wang, and X. J. Liu, "A wavelet numerical method for solving nonlinear fractional vibration, diffusion and wave equations," CMES: Computer Modeling in Engineering and Sciences, vol. 77, no. 2, pp. 137-160, 2011. 
[15] X. J. Liu, J. Z. Wang, X. M. Wang, and Y. H. Zhou, "Exact solutions of multi-term fractional diffusion-wave equations with Robin type boundary conditions," Applied Mathematics and Mechanics, vol. 35, no. 1, pp. 49-62, 2014.

[16] Y. Zhou, J. Wang, X. J. Zheng, and Q. Jiang, "Vibration control of variable thickness plates with piezoelectric sensors and actuators based on wavelet theory," Journal of Sound and Vibration, vol. 237, no. 3, pp. 395-410, 2000.

[17] J. Wang, X. Wang, and Y. Zhou, "A wavelet approach for activepassive vibration control of laminated plates," Acta Mechanica Sinica, vol. 28, no. 2, pp. 520-531, 2012.

[18] Ü. Lepik, "Solving fractional integral equations by the Haar wavelet method," Applied Mathematics and Computation, vol. 214, no. 2, pp. 468-478, 2009.

[19] Y. L. Li, N. Sun, B. C. Zheng, Q. Wang, and Y. C. Zhang, "Wavelet operational matrix method for solving the Riccati differential equation," Communications in Nonlinear Science and Numerical Simulation, vol. 19, no. 3, pp. 483-493, 2014.

[20] Y. H. Zhou and J. Z. Wang, "A generalized Gaussian integral method for the calculation of scaling function transforms of wavelets and its applications," Acta Mathematica Scientia A: Shuxue Wuli Xuebao, vol. 19, no. 3, pp. 293-300, 1999.

[21] J. Cang, Y. Tan, H. Xu, and S. Liao, "Series solutions of non-linear Riccati differential equations with fractional order," Chaos, Solitons and Fractals, vol. 40, no. 1, pp. 1-9, 2009.

[22] J. Z. Wang, Generalized theory and arithmetic of orthogonal wavelets and applications to researches of mechanics including piezoelectric smart structures [Ph.D. dissertation], Lanzhou University, 2001.

[23] S. H. Hosseinnia, A. Ranjbar, and S. Momani, "Using an enhanced homotopy perturbation method in fractional differential equations via deforming the linear part," Computers \& Mathematics with Applications, vol. 56, no. 12, pp. 3138-3149, 2008.

[24] Z. Odibat and S. Momani, "Modified homotopy perturbation method: application to quadratic Riccati differential equation of fractional order," Chaos, Solitons and Fractals, vol. 36, no. 1, pp. 167-174, 2008. 


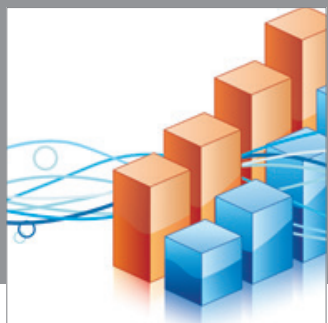

Advances in

Operations Research

mansans

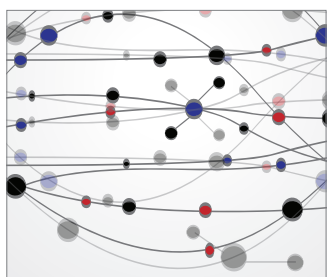

The Scientific World Journal
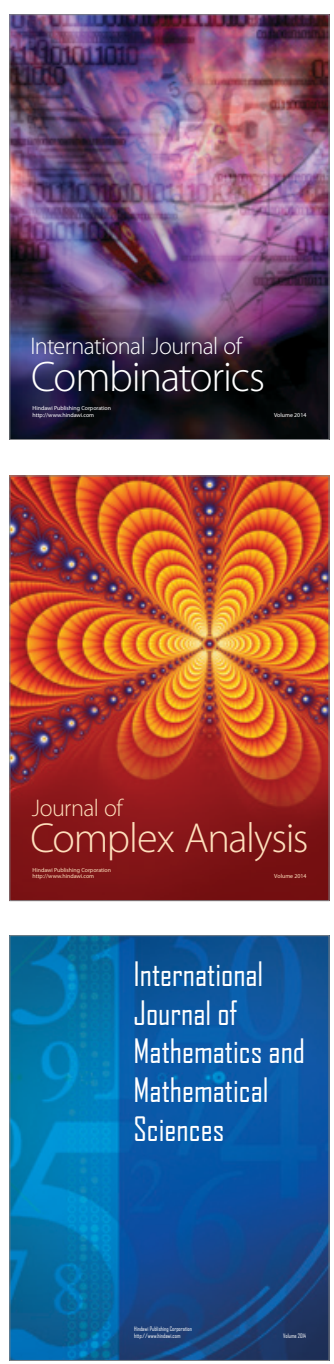
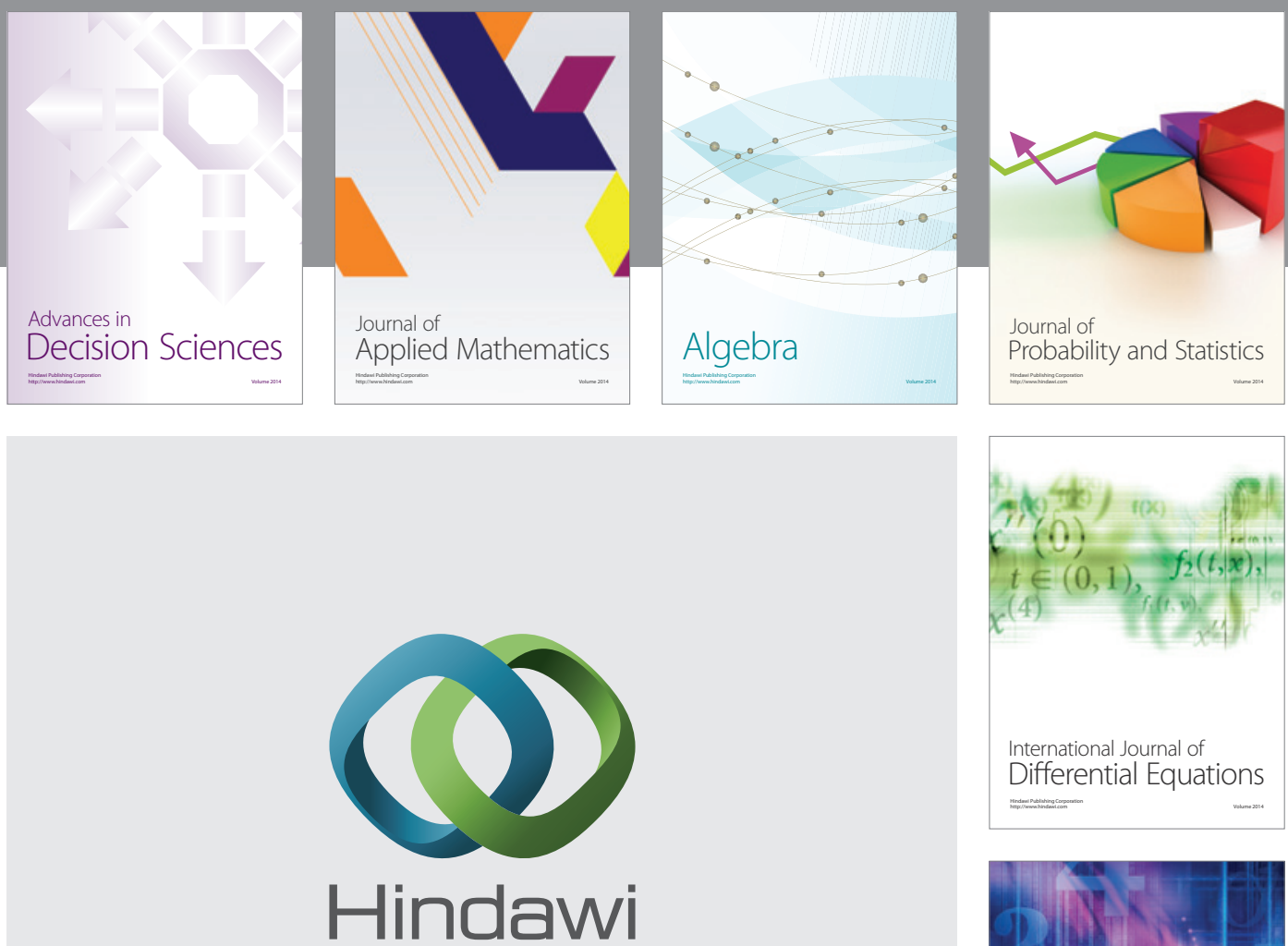

Submit your manuscripts at http://www.hindawi.com
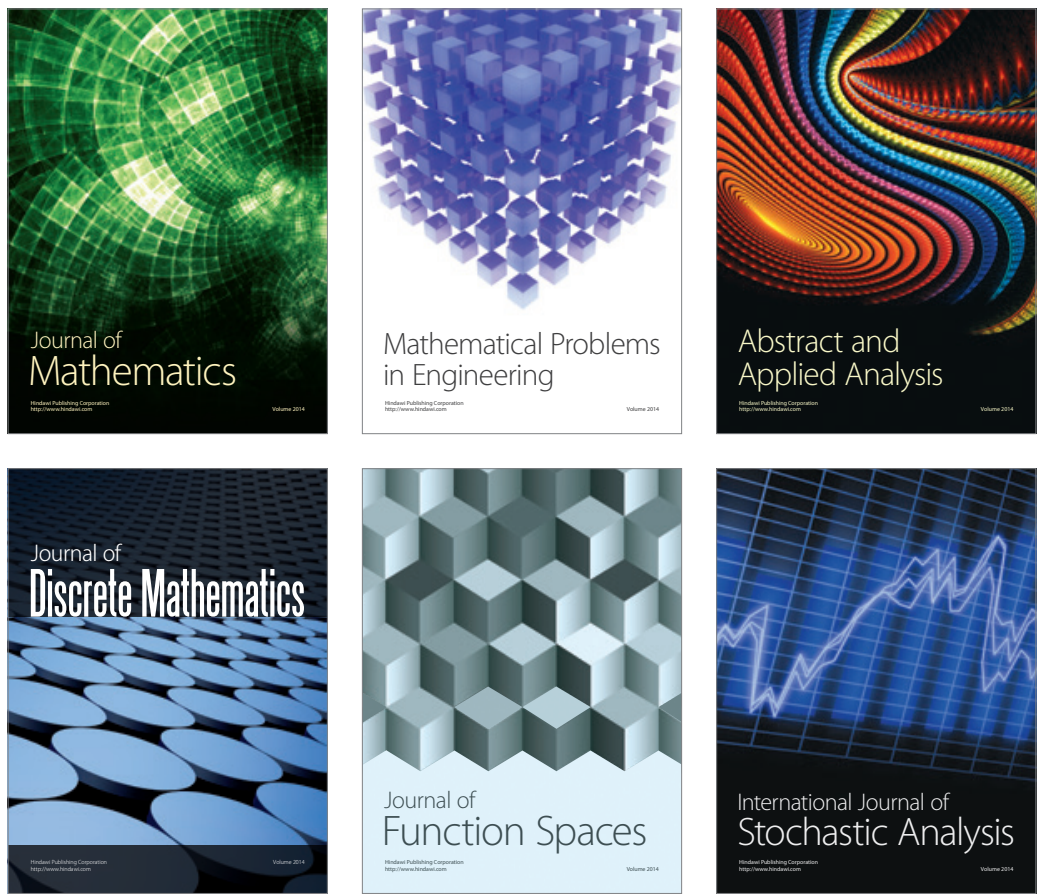

Journal of

Function Spaces

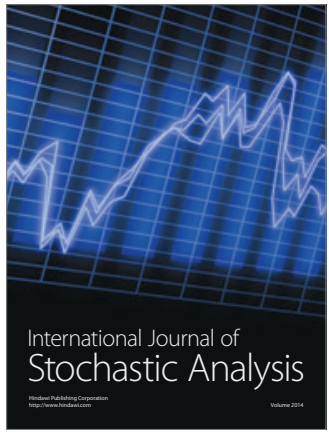

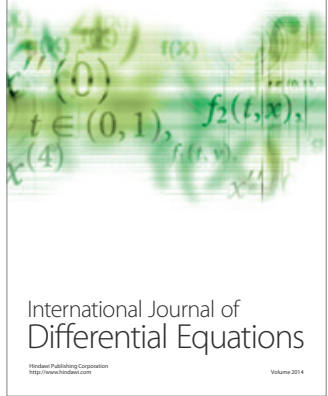
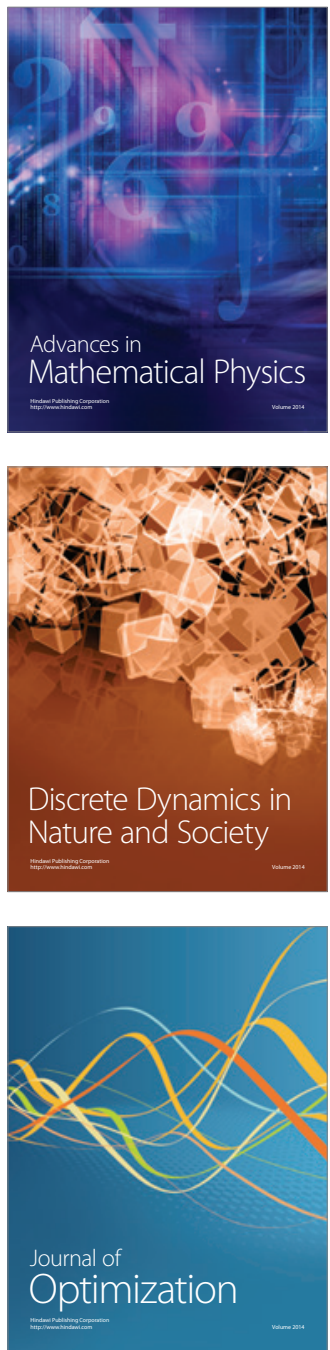